\title{
A Scalable Machine Learning System for Pre-Season Agriculture Yield Forecast
}

\author{
Igor Oliveira, Renato L. F. Cunha, Bruno Silva, Marco A. S. Netto \\ IBM Research
}

\begin{abstract}
Yield forecast is essential to agriculture stakeholders and can be obtained with the use of machine learning models and data coming from multiple sources. Most solutions for yield forecast rely on NDVI (Normalized Difference Vegetation Index) data which, besides being time-consuming to acquire and process, only allows forecasting once crop season has already started. To bring scalability for yield forecast, in the present paper we describe a system that incorporates satellite-derived precipitation and soil properties datasets, seasonal climate forecasting data from physical models and other sources to produce a pre-season prediction of soybean/maize yield-with no need of NDVI data. This system provides significantly useful results by the exempting the need for high-resolution remote-sensing data and allowing farmers to prepare for adverse climate influence on the crop cycle. In our studies, we forecast the soybean and maize yields for Brazil and USA, which corresponded to $44 \%$ of the world's grain production in 2016. Results show the error metrics for soybean and maize yield forecasts are comparable to similar systems that only provide yield forecast information in the first weeks to months of the crop cycle.
\end{abstract}

\section{INTRODUCTION}

Agriculture is an industry sector that is benefiting strongly from the development of sensor technology, data science, and machine learning $(\mathrm{ML})$ techniques in the latest years. These developments come to meet environmental and population pressures faced by our society, where reports indicate a need of strong global agriculture yield increase to provide food for a growing population in a warmer planet [1].

Yield forecast is one of the tasks that can be performed by current ML algorithms [2], [3], [4]. Field sensors, satellites, unmanned aerial vehicles (UAVs), and farming equipment can provide a significant amount of data on soil conditions, plant physiology, weather, climate, and several of the processes taking place in a farm. These datasets allow the creation of classification and forecast models that can be extremely helpful to agriculture production.

Most of the work done in the field of yield forecasting via ML makes use of some sort of remote sensing data over the farm, specially in the form of Normalized Difference Vegetation Index (NDVI) [5], a popular indicator of vegetation activity that can be retrieved from near-infrared and red spectral channels. These indexes have the advantage of providing direct observations of a farm and can be useful to follow the crop cycle. While these datasets provide insights on near real time to problems such as diseases and deficiencies, they allow yield forecasting only after planting occurs, as one can analyze crop development and try to predict its final outcome after harvesting.

In this paper, we introduce an ML-based system using data from multiple sources to perform soybean yield forecasting before the start of the crop season-process known as preseason forecasting. The system is composed of a recurrent neural network (RNN) trained with precipitation, temperature, and soil properties as features and historical observed soybean and/or maize yield at municipality level for $1500+$ cities in Brazil and USA as labels. We considered Brazil and the USA in our case studies as they are two of the largest crop producers of the world, accounting for $28 \%$ and $35 \%$ of soybean global production and $6 \%$ and $36 \%$ of maize global production respectively as of 2016 [6]. Operationally, the meteorological data is provided by a reanalysis-bases seasonal forecast product of temperature and precipitation, which allows for forecasting up to seven months in the future. Results are comparable and in some cases superior to similar models that need remote sensing data over the farm, thus only capable of providing a forecast in the first weeks/months of the crop cycle (early season forecast).

The two major contributions of this work are:

- A yield forecast system based on fewer data requirements compared to existing yield forecast solutions which demand large amounts of remote sensing data. Our system retrieves the necessary climate and soil properties data for a given coordinate automatically from an appropriate source. Another advantage is that the system works also on large regions, and provides forecasts at a resolution compatible with best input data resolution, which in the case is $250 \mathrm{~m}$ originally from the soil data.

- The capability of forecasting yield before the beginning of the crop season. This provides users the capability to perform strategy changes, like choosing a more robust genetic variation before planting or even changing the crop type, in order to accommodate for extreme climatic variations further ahead in the crop cycle.

The scalability for yield forecast comes from our observation that a neural network model can detect and exploit redundant information coming from soil and weather data. We also observed the neural network model can learn an implicit representation of the cycles of the crops, which is further detailed in this paper.

The paper is organized as follows. In Section II, we discuss existing work on yield forecast. We introduce the proposed 


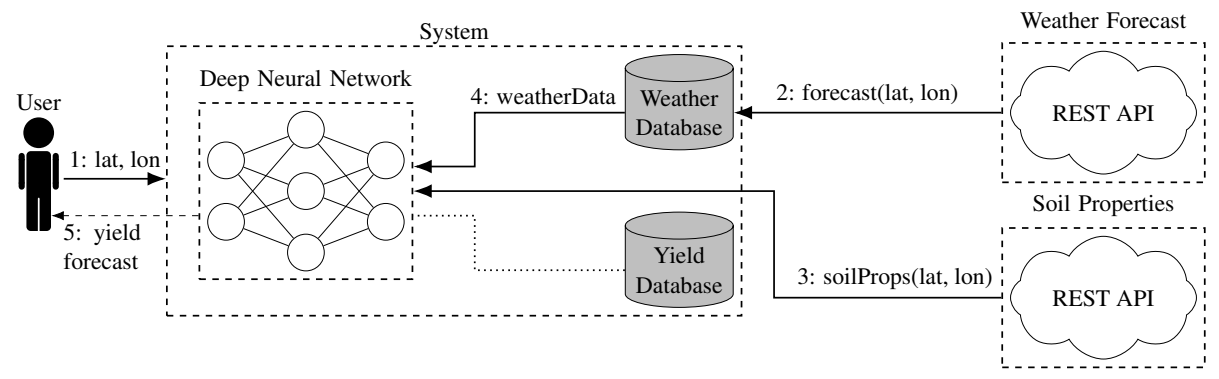

Fig. 1: System Architecture. The system we built can perform yield forecasts on the fly. It is able to do so by, upon user request (1), downloading cached weather forecast $(2,4)$ and soil properties data from RESTful APIs (3), and combining them to issue yield forecasts (5) using a trained deep neural network model. Both the APIs and the system work with bounding boxes for issuing yield forecasts for regions over the globe.

system containing the model RNN architecture with implementation justifications in Section III. In Section IV we present the system evaluation followed by conclusions in Section V.

\section{RELATED WORK}

Yield forecast is an important service of agriculture computational systems [7], [8]. In this section we cover a few efforts in this area, highlighting some with machine learning components.

Kogan et al. [9] compared different methods for winter wheat yield forecasting: using remote sensing observations, meteorological data and biophysical models. The two former methods consisted respectively of linear regression models using NDVI data at $250 \mathrm{~m}$ resolution and data from 180 weather stations for a 13-year period as predictors. The third method is based on the application of a biophysical processbased crop model, an algorithm that models phenology, canopy development, biomass accumulation, water stress and many other plant components. In this case, the World Food Studies (WOFOST) model [10] was used. All three approaches were used to perform forecast 2-3 months before harvest and the biophysical model showed the best results in terms of root mean squared error (RMSE). The NDVI-based and the meteorological data-based methods showed similar performance when minimum input data requirements were met.

In studying dryland maize in South Africa, Estes et al. [11] developed three empirical models that were compared against the CERES-maize model of the DSSAT platform [12]. Two of the empirical models were based on maximum entropy (MAXENT) [13]: one trained on all national crop distributions points and the other trained with the top producing localities. The third method used a generalized additive model (GAM) trained with yield data derived from NDVI. GAM and CERES results showed linear correlation to measured yield $\left(R^{2}=0.75\right.$ and 0.37 , respectively) as did the MAXENT model trained with high-productivity points $\left(\mathrm{R}^{2}=0.62\right)$.

Gonzalez-Sanchez et al. [14] compared the predictive accuracy of several techniques (Multiple linear regression, M5Prime regression trees, perceptron multilayer neural networks, support vector regression and $\mathrm{k}$-nearest-neighbors $/ \mathrm{KNN}$ ) for crop yield prediction in ten crop datasets from western Mexico.
Predictors came from typical atmospheric data (solar radiation, rainfall, temperature, etc) and some genetic and farm management information like season-duration cultivar and planting area. For these specific conditions, the regression trees and KNN showed the lowest error metrics.

Kumar [15] performed rice yield forecasting by adaptive neuro fuzzy inference system (ANFIS) technique. For that, they used 27 years time series data of yield and weather. ANFIS is an effort to integrate the benefits of neural networks and fuzzy logic in a single framework by using linguistic information from the fuzzy logic and learning capabilities of an artificial neural network (ANN). Quantitative performance assessment for rice and wheat yield observations in India showed good applicability of the technique in yield prediction.

Dahikar et al. [2] studied the basic requirements for applications of ANNs in yield prediction. Simple network architectures, with one hidden layer and back propagation of errors were tested for different predictors and crops, like cotton, sugarcane, wheat, rice and others. Soil parameters detected to be relevant for crop yield prediction were $\mathrm{PH}$ and concentrations of nitrogen, phosphate, potassium, organic carbon, calcium, magnesium, sulphur, manganese, copper and iron. In terms of atmospheric predictors, temperature, rainfall and humidity were the relevant features detected.

The proposed system differs from existing solutions as (i) it does not rely on NDVI data, which is a more scalable approach to handle country-level forecasts, and (ii) it is able to provide yield forecasts with a seven-month lead time, while offering similar (or even better) results to systems that perform short term yield forecasts.

\section{Architecture}

In this work, we combined atmospheric data (accumulated precipitation, maximum, and minimum temperature) and soil data to produce a model capable of generating yield forecast data—as illustrated in Figure 1. We implemented a Deep Neural Network (DNN) as our machine learning model. This section provides a description of the multiple data sources required to feed our DNN model, the model itself, and its usage in production. 


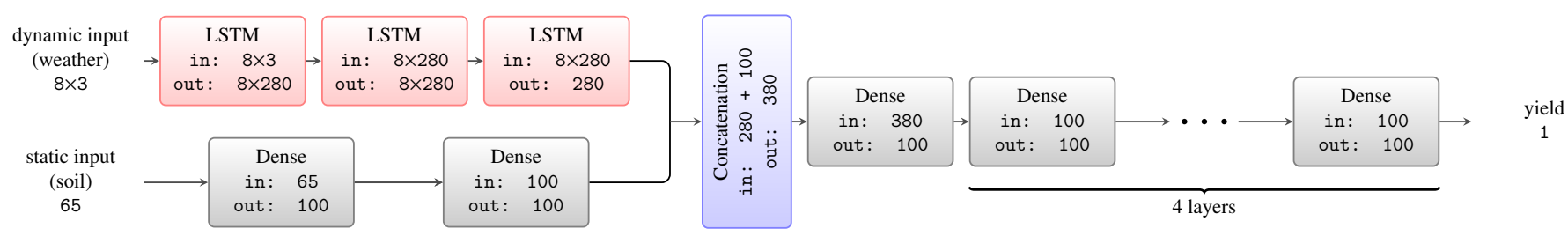

Fig. 2: DNN Architecture. Red nodes represent Long Short-Rerm Memory (LSTM) recurrent layers. Gray nodes represent dense, fully-connected layers. The blue node represents a concatenation layer, which concatenates the intermediate representations from the dynamic and static paths. Numbers below node names represent shapes of input and output tensors. For example, $\mathrm{n} \times \mathrm{m}$ represents a $\mathrm{n}$ by matrix, while single numbers represent line vectors.

\section{A. Data Sources}

Monthly precipitation data was provided by the Climate Hazards Group Infrared Precipitation with Stations (CHIRPS) dataset [16]. CHIRPS provides precipitation data at $0.05^{\circ}$ resolution by merging satellite and weather station information. CHIRPS uses satellite data in three ways: satellite means are used to produce high-resolution rainfall climatologies, infrared Cold Cloud Duration (CCD) fields are used to estimate monthly and, pentadal rainfall deviation from climatologies. Lastly, satellite precipitation fields are used to guide interpolation through local distance decay functions.

Monthly air temperature data, specifically minimum and maximum temperatures for each month, were provided by reanalysis datasets ${ }^{1}$ from ERA-Interim, one of the latest reanalysis products developed by the European Centre for MediumRange Weather Forecasts (ECMWF) [17]. This dataset covers the globe at a resolution of approximately $80 \mathrm{~km}$ per pixel and was generated using data assimilation from several sources. The project represents a significant improvement over past reanalysis efforts, due to advances in modeling of several climate processes such as the hydrological cycle and assimilation of cloud- and rain-affected satellite radiances.

Soil properties data comes from SoilGrids.org [18], an open, global soil dataset with a resolution of $250 \mathrm{~m}$ per pixel which provides information for clay, silt and sand contents plus fine earth and coarse fragments bulk density. All this data is available in seven depths $(0,5,15,30,60,100$ and $200 \mathrm{~cm})$. SoilGrids data are results of predictions based on 150000 soil profiles used for training and 158 remote sensing-based soil covariates. These were used to fit an ensemble of random forest, gradient boosting and multinomial logistic regression models.

As labels for training, the model uses actual yield data. In this work, we collected maize and/or soybean yield data at county or municipality level from each country's official agency. For Brazil, we used soybean data provided by IBGE (Brazil Statistics and Geography Bureau) [19], while for the USA we used maize and soybean data provided by the USDA (United States Department of Agriculture) [20]. Crop seasons in both countries last approximately six months, although the

\footnotetext{
${ }^{1}$ Reanalysis datasets are datasets produced both from observational data and numerical models.
}

TABLE I: Static features used in the Machine Learning model. All the soil features here are available in seven layers. Latitude and longitude of the prediction point are also included in the static inputs. Hence, there are $9 \times 7+2=65$ static features.

\begin{tabular}{lr}
\hline Feature & Unit \\
\hline Clay content (0-2 micrometer) mass fraction & {$[\%]$} \\
Silt content (2-50 micrometer) mass fraction & {$[\%]$} \\
Sand content (50-2000 micrometer) mass fraction & {$[\%]$} \\
Bulk density (fine earth) & {$[\mathrm{kg} / \mathrm{m} 3]$} \\
Coarse fragments volumetric & {$[\%]$} \\
Cation exchange capacity of soil & $\mathrm{cmolc} / \mathrm{kg}$ \\
Soil organic carbon content (fine earth fraction) & $\mathrm{g} \mathrm{per} \mathrm{kg}$ \\
Soil pH x 10 in H2O & - \\
Soil pH x 10 in KCl & - \\
Point Latitude & $\circ$ \\
Point Longitude & $\circ$ \\
\hline
\end{tabular}

tropical climate in Brazil allows for a second mini-season in-between main seasons. The dataset sizes used in training, validation, and testing are described in Table III. For each case, test sets were composed of $20 \%$ of the total data points, while training plus validation sets were composed of the remaining points (from which being 30\% validation and $70 \%$ for training).

We split the input data into two main categories: static for the soil data and dynamic for the weather data. The rationale behind this decision is that for the time scales considered in this work soil properties do not change over time, while meteorological data presents seasonal variability. Knowing the data we used has different characteristics allows us to approach them differently when building an ML model. The static features (corresponding to the soil data) we used to train the model are shown in Table I, and the dynamic features (corresponding to the atmospheric data) we used to train the model are shown in Table II.

\section{B. Neural Network Description}

As we split the data into dynamic and static sets, the data follow different pathways in the model before the joining the internal representations of both data types (Figure 2). For the static set, the data flows through a two-layer fullyconnected neural network before proceeding in the computational graph-each layer with a hundred hidden units. The dynamic set flows through a three-layer LSTM neural network, 
TABLE II: Dynamic Features used in the Machine Learning model. All the features in here are available for eight months covering the crop cycle in each geography (September to April in Brazil; April to October in US). Hence, there are $3 \times 8=24$ dynamic features.

\begin{tabular}{lr}
\hline Feature & Unit \\
\hline Minimum Temperature & {$\left[{ }^{\circ} \mathrm{C}\right]$} \\
Maximum Temperature & {$\left[{ }^{\circ} \mathrm{C}\right]$} \\
Precipitation & {$[\mathrm{mm}]$} \\
\hline
\end{tabular}

each with 280 memory cells and eight time steps (one for each month in the seasonal forecast), before proceeding in the computational graph. LSTM [21] units are recurrent neural network modules that are useful when dealing with data with a temporal relationship, and can learn to recognize temporally extended patterns in noisy sequences. Hence, they were chosen to model the dynamic input in our model.

The aforementioned describes the process outlined in the upper part of Figure 2 before the node labeled "Concatenation Layer". When both data paths are computed, the network joins them together through the concatenation of internal representations. After the concatenation, the network processes the joined data through five fully-connected layers, each with a hundred hidden units. Finally, the network outputs a single value: the forecasted yield. The model uses the Mean Absolute Error as cost function and uses scaled exponential linear units (SELUs) [22]. The SELU activation function is given by

$$
\operatorname{selu}(x)=\lambda\left\{\begin{array}{ll}
x & \text { if } x>0 \\
\alpha e^{x}-\alpha & \text { if } x \leq 0
\end{array} .\right.
$$

In Equation (1), $\alpha$ and $\lambda$ are chosen in a way that the mean and variance of the inputs are preserved between two consecutive layers. Hence, such an activation leads to Self-Normalizing Networks with the property of being robust to perturbations, and not having high variance in training errors [22].

A full description of the model as implemented in Keras [23] is shown in Figure 3 and represents everything that is needed to replicate the model described in this section, and ensures reproducibility of the results described in this work (Section IV-B).

\section{Production System}

As described in the previous section, after the DNN is fully trained, one can forecast the yield of a single point: given a pair $p=$ (latitude, longitude), one can query the meteorological and soil properties datasets, extract the data corresponding to $p$ and perform a forecast. Although this would work for any point in the globe, the model was trained with Brazil and US yield data so it wouldn't make practical sense to use it outside these geographies. Moreover, it is possible to integrate the DNN into a bigger system for on-the-fly yield forecast. We implemented the DNN as a component in a decision-support tool for agriculture. To forecast yields for future dates, we replaced the ERA-Interim data with seasonal forecasts (which are also based on reanalysis data) for the dynamic features

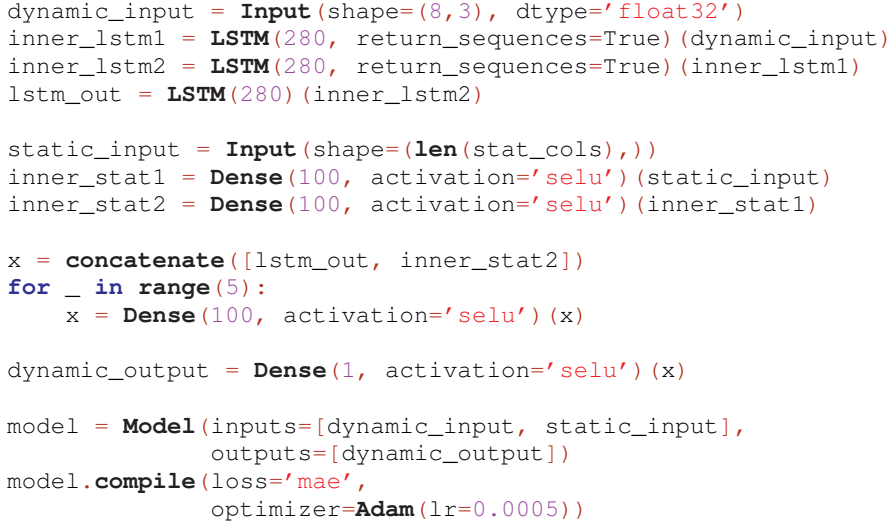

Fig. 3: DNN used in this work as a model implemented in Keras.

and continued using SoilGrids data. Due to its relatively high resolution, the SoilGrids data allows the system to also forecast with a resolution of $250 \mathrm{~m}$ per pixel.

\section{EVALUATION}

The goal of the evaluation is to demonstrate the effectiveness of the proposed yield forecast model against existing solutions that rely on remote sensing data, e.g. NDVI. We used five comparison metrics in the evaluation related to accuracy of the yield forecast.

\section{A. Experimental Setup}

Data for the 3 cases (US-Soybean, US-Maize and BrazilSoybean) was used in training and testing the DNN model. For each case, $80 \%$ of the total data points were selected as training set and the remaining $20 \%$ as test set. During training, $30 \%$ of the training set was selected for a validation set. All of these sets were randomly chosen, so the model could learn from many different scenarios of weather, soil and forms of growing. The sizes of each set are detailed in Table III.

Model training for each case took around 300 epochs until a minimum validation loss value was achieved. The evolution of training and validation losses is shown in Figure 4. Losses show a typical neural network behaviour of continuous decay for training loss, eventually decoupling from validation loss. The training made use of the Earlystopping callback function from the keras library, with a patience parameter (the number of epochs with no improvement after which training is stopped) equal to 50 . The DNN model could be successfully trained using a relatively modest GPU (Tesla $\mathrm{K} 40 \mathrm{~m})$ in reasonable time ( 10 minutes). Test sets for each geography and crop example were used for evaluation of the model performance in forecasting crop yield at pre-season time and are discussed in Section IV-B.

\section{B. Result Analysis}

The model was used to perform yield forecast for the points in the test set and the results shown in Table IV. Since the training and test sets were randomly selected from the 


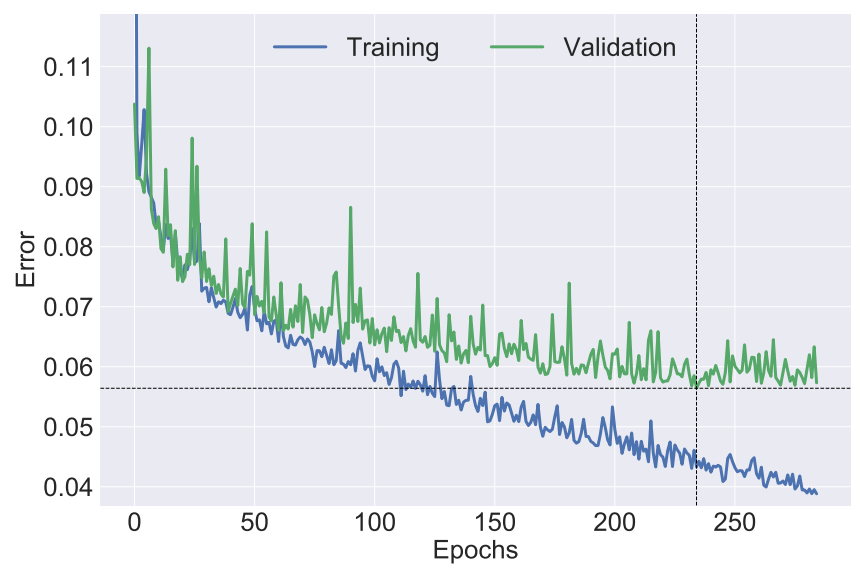

(a) US soybean model

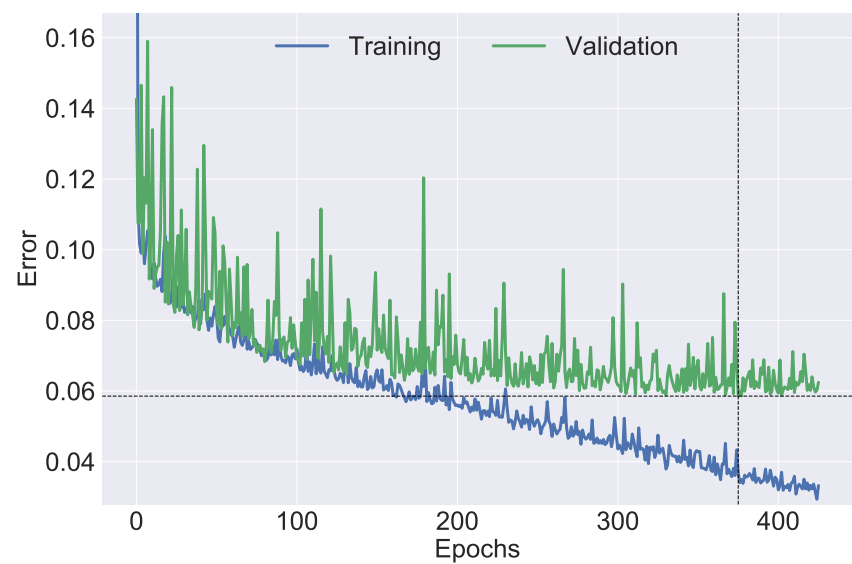

(b) US maize model

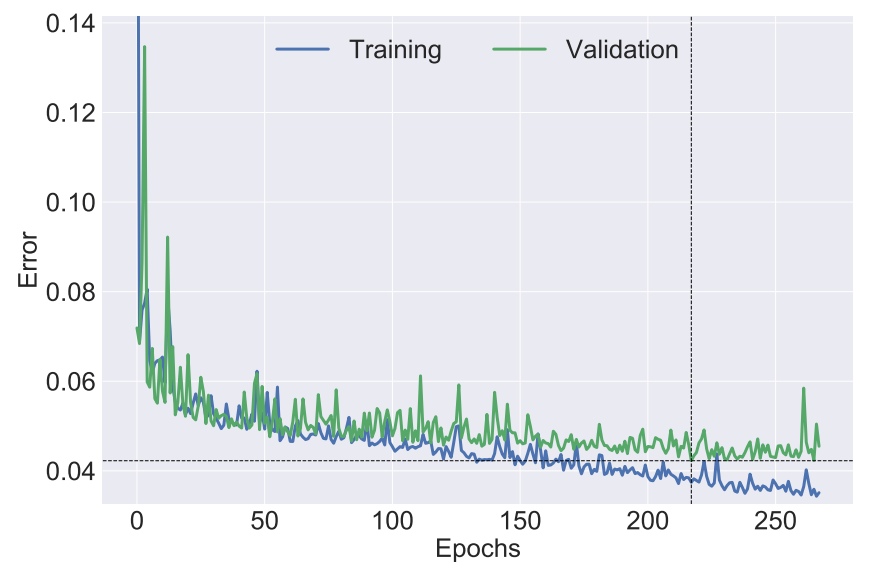

(c) Brazil soybean model

Fig. 4: Loss for training and validation sets across epochs for Brazil/USA soybean/maize models. Dashed lines indicate the chosen model, which is the one with minimum validation loss.

TABLE III: Datasets sizes used in training, testing and validation.

\begin{tabular}{lrrr}
\hline & Brazil-Soybean & US-Soybean & US-Maize \\
\hline \# of counties & 1529 & 1814 & 2204 \\
\# of data points & 16767 & 16939 & 19692 \\
Training set size & 9389 & 9485 & 11027 \\
Validation set size & 4023 & 4065 & 4725 \\
Test set size & 3354 & 3388 & 3819 \\
\hline
\end{tabular}

complete input dataset, a good performance in the test set indicates the model generalizes well for different seasonal climates, agriculture management practices, soil and other geographical characteristics. Figure 5 shows comparison of observed and forecasted yield for each geography and crop.

When comparing geographies, model results for the US are slightly better than the ones found for Brazil. It is important to notice the specific methodologies in gathering yield data for both countries. The former relies more on self-reporting information provided by farmers [19], whereas in the latter case there is a data gathering effort by the governmental agency [20]. These different methods are reflected on both raw datasets: the one for Brazil showed a higher number of missing information and in a relevant number of counties the reported yield remains unchanged along some years, which suggest the data is a gross estimation.

Model performance of the US-Soybean forecasts was superior to US-Maize. This initially indicates that the DNN architecture is better in creating a function that maps the physical relation between soil plus climate to yield in the soybean case. Physiological differences between soybean and maize determine the performance in each case. To account for the temperature influence on both crops, we can analyze the typical values of accumulated growing degree days (AGDD). Soybean typically has lower AGDD values (around 1100 ${ }^{\circ} \mathrm{C}$ ) [24] than Maize (around $1450{ }^{\circ} \mathrm{C}$ ) [25]. In terms of water needs, studies have showed maize has a higher vulnerability to moisture deficiency when compared to soybean [26]. All of these indicate that maize is more sensitive to the climate variables (temperature and precipitation) used in model training than soybean, meaning any uncertainties brought by the climate data input sources will have a stronger impact in the 


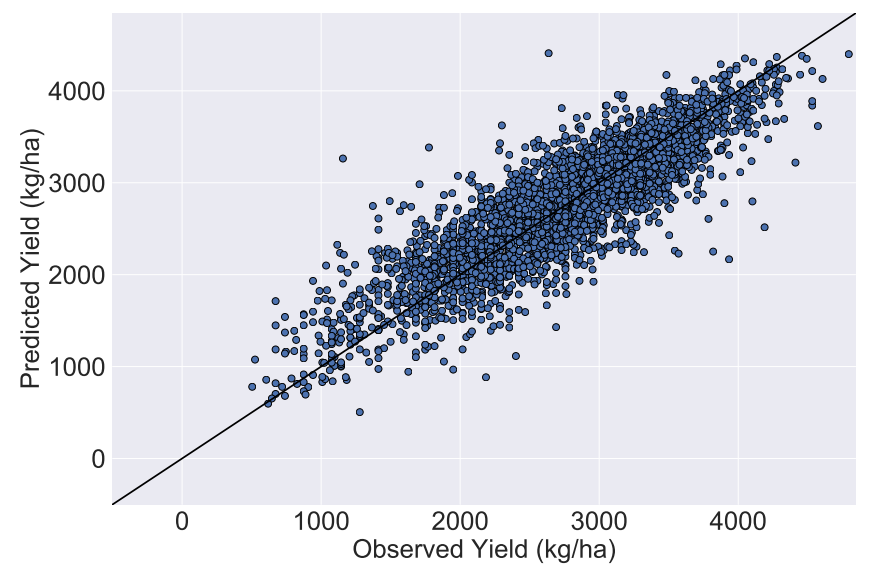

(a) US soybean model

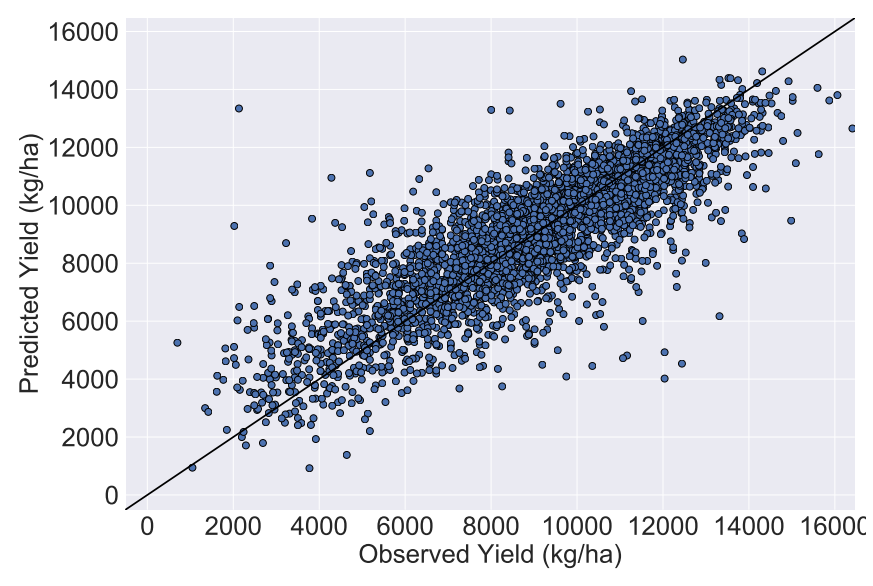

(b) US maize model

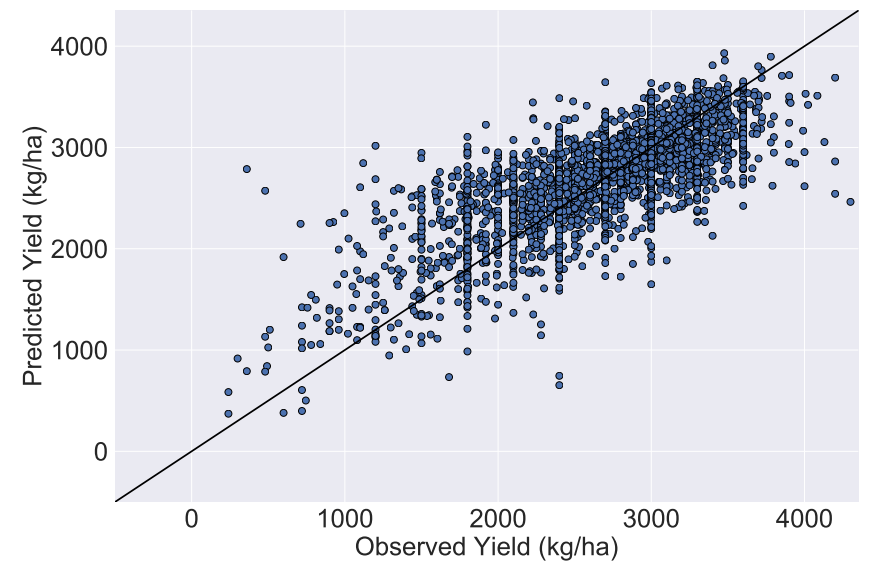

(c) Brazil soybean model

Fig. 5: Scatter plots of model performance according to country and crop.

maize yield forecast, which could explain the difference in performance of the model for the two crops.

The comparison of these results to other yield forecasting efforts should take in consideration several differences amongst these studies, including the selected statistics reported and its appropriate units. Metrics like the coefficient of determination $R^{2}$ and the ones that compute errors as a ratio of some average field (MAPE, RMSPE) can be compared across different crops and regions, besides being commonly reported. Table V shows some of these metrics reported in several studies. All of these efforts make use of some sort of remote-sensing, most frequently NDVI. The performance of this work is comparable with these studies but with a lot less data requirements and is able to provide useful information for agriculture operations before the seeding occurs.

\section{CONCLUSION}

Agriculture yield forecasts are a very useful tool for farm management and can help stakeholders to perform critical decisions in their agricultural operations. Many available methods provide yield forecast information, the vast majority of them through the use of some sort of remote sensing data
TABLE IV: Model scores.

\begin{tabular}{|c|c|c|c|}
\hline & Brazil - Soybean & US - Soybean & US - Maize \\
\hline $\mathrm{MAE}^{a}$ & 288.39 & 270.18 & 1031.00 \\
\hline $\mathrm{MAPE}^{b}$ & $10.70 \%$ & $9.80 \%$ & $11.31 \%$ \\
\hline $\mathrm{RMSE}^{c}$ & 385.81 & 354.08 & 1393.02 \\
\hline $\mathrm{RMSPE}^{d}$ & $14.31 \%$ & $12.85 \%$ & $15.28 \%$ \\
\hline $\mathrm{R}^{2 e}$ & 0.55 & 0.75 & 0.71 \\
\hline & & $\begin{array}{r}{ }^{a} \text { Mear } \\
\text { Mean Absolute } \\
{ }_{c} \text { Root Me } \\
\text { ot Mean Square I } \\
{ }^{e} \text { Coefficient }\end{array}$ & $\begin{array}{l}\text { Absolute Erro } \\
\text { rcentage Erro } \\
\text { n Square Erro } \\
\text { rcentage Erro } \\
\text { determinatior }\end{array}$ \\
\hline
\end{tabular}

(like NDVI) from the farm fields. While this allows for highresolution forecasts, it comes with the high cost of acquiring and processing these extra datasets, which can be relevant depending on the properties dimensions.

Machine-learning is one of the techniques gaining popularity for agriculture applications, specially with the increasing number of new data sources being developed in the latest years. We propose a machine learning system that provides pre-season yield forecasting, meaning farmers can make farm 
TABLE V: Comparison of yield forecasting methods to the ML system proposed in this work.

\begin{tabular}{|c|c|c|c|c|c|}
\hline Study & Crop & $\mathrm{R}_{\alpha}^{2}$ & $\operatorname{MAPE}_{\beta}[\%]$ & $\operatorname{RMSPE}_{\gamma}[\%]$ & ML System \\
\hline Kolotii, A et al., 2015 [27] & Wheat & $0.50-0.80$ & - & - & $0.55-0.75_{\alpha}$ \\
\hline Capa-Morocho, M et al., 2016 [28] & Wheat, Maize & - & - & $2.1-13.2$ & $\mathrm{NA}-15.28 \gamma$ \\
\hline Meroni, M et al., 2016 [29] & Grain crops & $0.62-0.91$ & - & - & $0.55-0.75_{\alpha}$ \\
\hline Morell, F et al., 2016 [30] & Maize & - & - & $20-34$ & $2.85-15.28 \gamma$ \\
\hline Johnson, D 2014 [31] & Maize, Soybean & $0.47-0.77$ & - & - & $0.55-0.75_{\alpha}$ \\
\hline Pagani, V et al., 2018 [32] & Rice & $0.21-0.89$ & - & - & $0.55-0.75_{\alpha}$ \\
\hline Kumar, N et al., 2014 [33] & Rice, Wheat & $0.53-0.58$ & - & - & $0.55-0.75_{\alpha}$ \\
\hline Bose, P et al., 2016 [34] & Wheat & & $0.13-27.97$ & - & $9.8-11.31_{\beta}$ \\
\hline Satir, O and Berberoglu, S, 2016 [35] & Maize, Wheat, Cotton & $0.50-0.67$ & $6.30-7.30$ & - & $0.55-0.75_{\alpha} \& 9.8-11.31_{\beta}$ \\
\hline
\end{tabular}

management decisions (like choosing different crops or genetic variations) before seeding occurs.

The system proposed in this work is formed by a neural network where inputs are treated separately. Static soil data in handled by fully-connected layers while dynamic meteorological data is handled by recurrent LSTM layers. This particular architecture was trained with historical data for several soil properties, precipitation, minimum and maximum temperature against historical yield labels at county level for two crops (maize and soybean) and two geographies (Brazil and US), which production correspond to $44 \%$ of the global grain production [6]. After training, the model was tested in a separate dataset and showed comparable results with existing yield forecasting methods that make use of extensive remotesensing data. The major lesson learnt from our experiments is that it is possible obtain scalable yield forecast because the proposed neural network model can detect and exploit redundant information both in the soil and in the weather data. Additionally, the model may have been able to learn an implicit representation of the cycles of the crops evaluated in this paper, considering the seasonal atmospheric data used as input.

Our results show that farmers and agriculture stakeholders can benefit from useful information with significantly fewer data requirements and maintain useful accuracy values. The global availability of the input datasets also allows the system to easily scale across different regions if local yield data is present. Although the used input datasets allow for relatively high-resolution forecasts $(250 \mathrm{~m})$, the existing system can be used as a foundation for future precision agriculture services by assimilating more traditional NDVI and similar datasets.

\section{REFERENCES}

[1] I. FAO, "Wfp (2015), the state of food insecurity in the world 2015 meeting the 2015 international hunger targets: taking stock of uneven progress," Food and Agriculture Organization Publications, Rome, 2016.

[2] S. S. Dahikar and S. V. Rode, "Agricultural crop yield prediction using artificial neural network approach," International Journal of Innovative Research in Electrical, Electronics, Instrumentation and Control Engineering, vol. 2, no. 1, pp. 683-686, 2014.

[3] B. Ji, Y. Sun, S. Yang, and J. Wan, "Artificial neural networks for rice yield prediction in mountainous regions," The Journal of Agricultural Science, vol. 145, no. 3, pp. 249-261, 2007.

[4] A. X. Wang, C. Tran, N. Desai, D. Lobell, and S. Ermon, "Deep transfer learning for crop yield prediction with remote sensing data," in Proceedings of the 1st ACM SIGCAS Conference on Computing and Sustainable Societies. ACM, 2018, p. 50.
[5] A. Van de Griend and M. Owe, "On the relationship between thermal emissivity and the normalized difference vegetation index for natural surfaces," International Journal of remote sensing, vol. 14, no. 6, pp. $1119-1131,1993$.

[6] F. FAO and A. O. of the United Nations, "Faostat database," Available at: http://www.fao.org/faostat/en/ (accessed 13.06.18), 2016

[7] D. P. Holzworth, V. Snow, S. Janssen, I. N. Athanasiadis, M. Donatelli, G. Hoogenboom, J. W. White, and P. Thorburn, "Agricultural production systems modelling and software: current status and future prospects," Environmental Modelling \& Software, vol. 72, pp. 276-286, 2015.

[8] K. Louhichi, S. Janssen, A. Kanellopoulos, H. Li, N. Borkowski, G. Flichman, H. Hengsdijk, P. Zander, M. B. Fonseca, G. Stokstad et al., "A generic farming system simulator," in Environmental and Agricultural Modelling. Springer, 2010, pp. 109-132.

[9] F. Kogan, N. Kussul, T. Adamenko, S. Skakun, O. Kravchenko, O. Kryvobok, A. Shelestov, A. Kolotii, O. Kussul, and A. Lavrenyuk, "Winter wheat yield forecasting in ukraine based on earth observation, meteorological data and biophysical models," International Journal of Applied Earth Observation and Geoinformation, vol. 23, pp. 192-203, 2013.

[10] H. Boogaard, C. Van Diepen, R. Rotter, J. Cabrera, and H. Van Laar, "Wofost 7.1; user's guide for the wofost 7.1 crop growth simulation model and wofost control center 1.5," SC-DLO, Tech. Rep., 1998.

[11] L. Estes, B. Bradley, H. Beukes, D. Hole, M. Lau, M. Oppenheimer, R. Schulze, M. Tadross, and W. Turner, "Comparing mechanistic and empirical model projections of crop suitability and productivity: implications for ecological forecasting," Global Ecology and Biogeography, vol. 22, no. 8, pp. 1007-1018, 2013.

[12] J. W. Jones, G. Hoogenboom, C. H. Porter, K. J. Boote, W. D. Batchelor, L. Hunt, P. W. Wilkens, U. Singh, A. J. Gijsman, and J. T. Ritchie, "The dssat cropping system model," European journal of agronomy, vol. 18, no. 3-4, pp. 235-265, 2003.

[13] S. J. Phillips, R. P. Anderson, and R. E. Schapire, "Maximum entropy modeling of species geographic distributions," Ecological modelling, vol. 190 , no. 3-4, pp. 231-259, 2006.

[14] A. Gonzalez-Sanchez, J. Frausto-Solis, and W. Ojeda-Bustamante, "Predictive ability of machine learning methods for massive crop yield prediction," Spanish Journal of Agricultural Research, vol. 12, no. 2, pp. 313-328, 2014.

[15] P. Kumar, "Crop yield forecasting by adaptive neuro fuzzy inference system," Mathematical Theory and Modeling, vol. 1, no. 3, pp. 1-7, 2011.

[16] C. Funk, P. Peterson, M. Landsfeld, D. Pedreros, J. Verdin, S. Shukla, G. Husak, J. Rowland, L. Harrison, A. Hoell et al., "The climate hazards infrared precipitation with stations-a new environmental record for monitoring extremes," Scientific data, vol. 2, p. 150066, 2015.

[17] D. P. Dee, S. Uppala, A. Simmons, P. Berrisford, P. Poli, S. Kobayashi, U. Andrae, M. Balmaseda, G. Balsamo, d. P. Bauer et al., "The erainterim reanalysis: Configuration and performance of the data assimilation system," Quarterly Journal of the royal meteorological society, vol. 137, no. 656, pp. 553-597, 2011.

[18] T. Hengl, J. M. de Jesus, G. B. Heuvelink, M. R. Gonzalez, M. Kilibarda, A. Blagotić, W. Shangguan, M. N. Wright, X. Geng, B. BauerMarschallinger et al., "Soilgrids250m: Global gridded soil information based on machine learning," PLoS one, vol. 12, no. 2, p. e0169748, 2017.

[19] I. B. de Geografia e Estatística (IBGE), "Produção agrícola municipal," http://www.ibge.gov.br, 2016 
[20] U. NASS, "United states department of agriculture-national agricultural statistics service," Cropland Data Layer, 2018.

[21] S. Hochreiter and J. Schmidhuber, "Long short-term memory," Neural computation, vol. 9, no. 8, pp. 1735-1780, 1997.

[22] G. Klambauer, T. Unterthiner, A. Mayr, and S. Hochreiter, "Selfnormalizing neural networks," in Advances in Neural Information Processing Systems, 2017, pp. 972-981.

[23] F. Chollet et al., "Keras," https://github.com/fchollet/keras, 2015.

[24] F. A. Akyuz, H. Kandel, and D. Morlock, "Developing a growing degree day model for north dakota and northern minnesota soybean," Agricultural and Forest Meteorology, vol. 239, pp. 134-140, 2017.

[25] B. Martin, C. Dillon, T. Mark, T. Davis et al., "Determining the optimal decision to store or contract sale food-grade corn, field corn, and soybeans," in 2017 Annual Meeting, February 4-7, 2017, Mobile, Alabama, no. 252764. Southern Agricultural Economics Association, 2017.

[26] S. C. Zipper, J. Qiu, and C. J. Kucharik, "Drought effects on us maize and soybean production: spatiotemporal patterns and historical changes," Environmental Research Letters, vol. 11, no. 9, p. 094021, 2016.

[27] A. Kolotii, N. Kussul, A. Shelestov, S. Skakun, B. Yailymov, R. Basarab, M. Lavreniuk, T. Oliinyk, and V. Ostapenko, "Comparison of biophysical and satellite predictors for wheat yield forecasting in ukraine," The International Archives of Photogrammetry, Remote Sensing and Spatial Information Sciences, vol. 40, no. 7, p. 39, 2015.

[28] M. Capa-Morocho, A. V. Ines, W. E. Baethgen, B. Rodríguez-Fonseca, E. Han, and M. Ruiz-Ramos, "Crop yield outlooks in the iberian peninsula: Connecting seasonal climate forecasts with crop simulation models," Agricultural Systems, vol. 149, pp. 75-87, 2016.
[29] M. Meroni, D. Fasbender, R. Balaghi, M. Dali, M. Haffani, I. Haythem, J. Hooker, M. Lahlou, R. Lopez-Lozano, H. Mahyou et al., "Evaluating ndvi data continuity between spot-vegetation and proba-v missions for operational yield forecasting in north african countries," IEEE Transactions on Geoscience and Remote Sensing, vol. 54, no. 2, pp. 795-804, 2016.

[30] F. J. Morell, H. S. Yang, K. G. Cassman, J. Van Wart, R. W. Elmore, M. Licht, J. A. Coulter, I. A. Ciampitti, C. M. Pittelkow, S. M. Brouder et al., "Can crop simulation models be used to predict local to regional maize yields and total production in the us corn belt?" Field crops research, vol. 192, pp. 1-12, 2016.

[31] D. M. Johnson, "An assessment of pre-and within-season remotely sensed variables for forecasting corn and soybean yields in the united states," Remote Sensing of Environment, vol. 141, pp. 116-128, 2014.

[32] V. Pagani, T. Guarneri, L. Busetto, L. Ranghetti, M. Boschetti, E. Movedi, M. Campos-Taberner, F. J. Garcia-Haro, D. Katsantonis, D. Stavrakoudis et al., "A high-resolution, integrated system for rice yield forecasting at district level," Agricultural Systems, 2018.

[33] N. Kumar, R. Pisal, S. Shukla, and K. Pandey, "Crop yield forecasting of paddy, sugarcane and wheat through linear regression technique for south gujarat," Mausam, vol. 65, no. 3, pp. 361-364, 2014.

[34] P. Bose, N. K. Kasabov, L. Bruzzone, and R. N. Hartono, "Spiking neural networks for crop yield estimation based on spatiotemporal analysis of image time series," IEEE Transactions on Geoscience and Remote Sensing, vol. 54, no. 11, pp. 6563-6573, 2016.

[35] O. Satir and S. Berberoglu, "Crop yield prediction under soil salinity using satellite derived vegetation indices," Field crops research, vol. 192, pp. 134-143, 2016. 